\title{
Granulocyte-macrophage colony stimulating factor exacerbates collagen induced arthritis in mice
}

\author{
Ian K Campbell, Alison Bendele, David A Smith, John A Hamilton
}

\begin{abstract}
Objective-To examine the effect of granulocyte-macrophage colony stimulating factor (GM-CSF) on disease progression in the collagen induced arthritis (CIA) model in mice.

Methods-DBA/1 mice were primed for a suboptimal CIA response by intradermal injection of chick type II collagen without a secondary immunisation. Three weeks after immunisation the mice were given four to five consecutive daily intraperitoneal injections of recombinant murine GM-CSF $\left(15 \mu \mathrm{g} ; 5 \times 10^{5} \mathrm{U}\right)$, or vehicle, and arthritis development was monitored by clinical scoring of paws and calliper measurements of footpad swelling. At approximately six to eight weeks after immunisation mice were killed, their limbs removed and processed for histological analyses of joint pathology.

Results-Control animals receiving a single immunisation with collagen exhibited a varied CIA response both in terms of incidence and severity. Mice treated with GM-CSF at 20 to 25 days after immunisation with collagen had a consistently greater incidence and more rapid onset of disease than the vehicle treated control mice, based on clinical assessment. GM-CSF treated mice showed higher average clinical scores and greater paw swelling than controls. Histological analyses of joints reflected the clinical scores with GM-CSF treated mice displaying more pronounced pathology (synovitis, pannus formation, cartilage and bone damage) than control mice.
\end{abstract}

Conclusion-GM-CSF is a potent accelerator of the pathological events leading to chronic inflammatory polyarthritis in murine CIA supporting the notion that GM-CSF may play a part in inflammatory polyarthritis, such as rheumatoid arthritis.

(Ann Rheum Dis 1997;56:364-368)

The colony stimulating factors (CSFs) are glycoproteins believed to be essential for the survival, proliferation, and differentiation of haemopoietic progenitor cells into monocyte/ macrophages and granulocytes (see Metcalf for review $^{1}$ ). They include granulocytemacrophage CSF (GM-CSF), granulocyte CSF (G-CSF), macrophage CSF (M-CSF or $\mathrm{CSF}-1$ ), and interleukin 3 (IL3, or
multi-CSF), each differing in its progenitor cell specificity and biochemical properties. Although apparently necessary for normal haemopoiesis, there is evidence that they may have other functions as they can act on mature cells in the haemopoietic cell lineages. For example, GM-CSF could play a part in inflammation and immune reactions based on a number of in vitro reactions. ${ }^{1}$ These actions include induction of monocyte/macrophage class II MHC expression and development of antigen presenting (dendritic) cells, stimulation of monocyte/macrophage plasminogen activator production, enhancement of monocyte and granulocyte cellular adherence, augmentation of antigen presenting function of adherent spleen cells, priming of monocytes for cytokine production, stimulation of phagocytosis and superoxide production by neutrophils as well as being chemotactic for these cells. In addition, GM-CSF transgenic mice have certain lesions, which are probably explained by increased local macrophage numbers and functional capacity. ${ }^{2}$

Rheumatoid arthritis (RA) is an inflammatory joint disease of unknown aetiology for which there is evidence GM-CSF may be involved. GM-CSF has been identified in the joint effusions of RA patients, ${ }^{3}$ GM-CSF receptor mRNA expression has been observed in both rheumatoid and osteoarthritic synovia by polymerase chain reaction analysis ${ }^{4}$ and increased percentages of GM-CSF receptor bearing monocytes in the blood of RA patients compared with healthy people has been described. ${ }^{5}$ GM-CSF, when given to patients with Felty's syndrome for correction of neutropenia, can cause a flare up of the RA in the affected joints. ${ }^{6}$ Our own in vitro studies ${ }^{78}$ have shown that resident joint cells (chondrocytes and synovial fibroblasts) produce GM-CSF in culture in response to interleukin 1 (IL1) and tumour necrosis factor $\alpha(\mathrm{TNF} \alpha)$. Such findings, together with the finding that GM-CSF can prime monocyte/macrophages for the production of these and other proinflammatory mediators, led to the proposal of a CSF network loop in RA ${ }^{9}$ where GM-CSF has a central role in maintaining joint inflammation.

Animal models of arthritis provide a useful means of assessing in a controlled and defined way the importance of cytokines and other mediators in contributing to disease pathogenesis. In particular, collagen induced arthritis (CIA) in mice has been extensively used as a
Accepted for publication 3 April 1997 
model for human RA, with which it has many immunological and pathological parallels (see Staines and Wooley for review ${ }^{10}$ ). The purpose of this study was to obtain further evidence for a role for GM-CSF in the pathogenesis of arthritis by examining the effect of exogenous GM-CSF in CIA by direct cytokine injection. We report that recombinant murine GM-CSF increased the severity of CIA in DBA/ 1 mice given a single immunisation with collagen, as assessed both clinically and histologically.

\section{Methods}

REAGENTS

Chick type II collagen (CII) and Freund's incomplete adjuvant (FIA) were purchased from the Sigma Chemical Co (St Louis, MO); heat killed Mycobacterium tuberculosis (H37 Ra) was purchased from Difco Laboratories (Detroit, MI). Recombinant murine GM-CSF (specific activity $3 \times 10^{7} \mathrm{U} / \mathrm{mg}$, where $1 \mathrm{U}$ gives $1 / 2$ maximal stimulation of murine bone marrow cell colony formation) was provided by Amgen.

ANIMALS

Male DBA/1 mice were purchased from the Animal Resources Centre (Canning Vale, Western Australia) and allowed to acclimatise for one week before experimentation at which time they were aged 8 to 12 weeks. Animals were fed standard rodent chow and water ad libitum and housed in sawdust lined cages in groups of five.

\section{INDUCTION OF CIA}

Chick CII was dissolved at $2 \mathrm{mg} / \mathrm{ml}$ in $10 \mathrm{mM}$ acetic acid by end over end rotation overnight at $4^{\circ} \mathrm{C}$. An emulsion was prepared by mixing the collagen solution 1:1 with Freund's complete adjuvant prepared by supplementing FIA with $5 \mathrm{mg} / \mathrm{ml} M$ tuberculosis. Mice were injected intradermally at several sites into the base of the tail with a total of $100 \mu \mathrm{l}$ emulsion containing $100 \mu \mathrm{g}$ CII. After three weeks mice were given four to five daily intraperitoneal (ip) injections of either GM-CSF $\left(15 \mu \mathrm{g} ; 5 \times 10^{5} \mathrm{U}\right)$ or the same volume of vehicle.

\section{ASSESSMENT OF ARTHRITIS}

Animals were assessed for redness and swelling of limbs and a clinical score allocated for each mouse two to three times per week, for up to 58 days. The scoring system was based on that of Williams et $a l^{11}$ where $0=$ normal, $1=$ slight swelling and/or erythema, $2=$ extensive swelling and/or erythema and $3=$ joint distortion and/or rigidity. The maximum score per mouse was 12. Mice were considered to have arthritis when two consecutive positive evaluations were obtained. In some instances only individual digits were observed to swell on a particular limb in which case the maximum score assigned was 1 , regardless of the degree of swelling or stiffness of the $\operatorname{digit}(\mathrm{s})$. Paw swelling was also measured with callipers (Mitutoyo, Japan), accurate to $0.01 \mathrm{~mm}$.
HISTOLOGICAL EXAMINATION

At death, all limbs were removed, fixed in $4 \%$ paraformaldehyde for at least two days, and decalcified in Surgipath Decalcifier I (Surgipath, Grayslake, IL) for four days. The joints (fore paw, knee, ankle, and hind paw) were processed for paraffin wax embedding and $5 \mu \mathrm{m}$ sections prepared and stained with haematoxylin and eosin or toluidine blue. The sections were evaluated without knowledge of the treatment groups. Each of the above listed joints was scored on a 0 to 5 basis (with 0 being normal and 5 being severely affected) for the histological changes of inflammation (both synovitis and periarticular), pannus formation, cartilage damage (marginal erosions as well as diffuse changes), and bone damage (resorption, periosteal proliferation), as previously described. ${ }^{12}$ In the case of the paws, where numerous phalangeal and metacarpal or metatarsal joints were evaluated and scored concurrently, if only one to two joints of a paw were affected, the maximum score was 2 for any parameter, regardless of the severity of those affected joints. If three or more joints were affected the score reflected overall severity.

STATISTICAL ANALYSIS

The Mann-Whitney two sample rank test was used to determine the level of significance between means of groups. The incidence of arthritis between different groups was assessed by the $\chi^{2}$ test. For each test $\mathrm{p}<0.05$ was considered statistically significant.

\section{Results}

MICE TREATED WITH GM-CSF EXHIBIT MORE SEVERE ARTHRITIS

The onset and progression of arthritis was assessed by both clinical scores, based on redness and swelling of joints, as well as by calliper measurements of footpad thickness. DBA/ 1 mice were primed for suboptimal development of CIA by single immunisation with chick CII; the secondary immunisation usually performed in conventional CIA and resulting in greater incidence and severity of disease was omitted. The purpose of this was to present a model in which the actions of exogenously added proinflammatory cytokines might be more evident, as previously described. ${ }^{12}$ Under these conditions, the first clinical signs of arthritis usually appeared by approximately day 30 . We observed the arthritis in this model to manifest itself in a spectrum of responses ranging from the swelling of single or multiple digits only to generalised paw swelling, with and without inclusion of the digits.

In view of the proinflammatory actions of GM-CSF ${ }^{13}$ and its presence in rheumatoid joint effusions ${ }^{3}$ it was of interest to examine the effect of GM-CSF administration in this model. At three weeks after immunisation with CII, mice were given four to five consecutive, daily ip injections of GM-CSF. The injection schedule was based on similar studies examining the effect of IL1 in this model ${ }^{12}$ and is considered to be at a time when there is an established immune response to CII but before the onset of disease. Mice so treated with 
Table 1 Effect of GM-CSF on incidence and average clinical score of CIA *

\begin{tabular}{|c|c|c|c|c|c|c|}
\hline \multirow[b]{2}{*}{$G M-C S F$} & \multirow[b]{2}{*}{ Days } & \multirow[b]{2}{*}{ Killt } & \multicolumn{2}{|c|}{ Incidence (n) } & \multicolumn{2}{|c|}{ Average clinical score $\neq$} \\
\hline & & & - & + & - & + \\
\hline Experiment 1 & $21-25$ & 45 & $2 / 10$ & $7 / 10$ & $0.1(0.1)$ & $1.3(0.5)$ \\
\hline Experiment 2 & $20-23$ & 43 & $9 / 10$ & $8 / 8$ & $1.2(0.3)$ & $3.4(0.7) \Phi$ \\
\hline Experiment 3 & $21-24$ & 58 & $7 / 10$ & $10 / 10$ & $1.4(0.5)$ & $3.4(0.6) \S$ \\
\hline
\end{tabular}

ॠ DBA/1 mice, immunised with chick CII, were given daily ip injections at different times (see Days) with either $15 \mu \mathrm{g}$ GM-CSF (+) or vehicle (-) and the arthritis assessed by clinical score

every two to three days. $\dagger$ Day of death. $¥$ The average clinical score of each mouse was
determined for the period immediately following GM-CSF, or vehicle treatment, to death.

Values show the mean (SEM) of these values for the groups of mice. $\S p<0.05$ compared with

vehicle control. (experiment 1 from table 1). There was a strong concordance between the clinical and histological assessments for individual limbs. For each parameter the joints from GM-CSF treated mice scored significantly higher than the joints of control mice, consistent with the

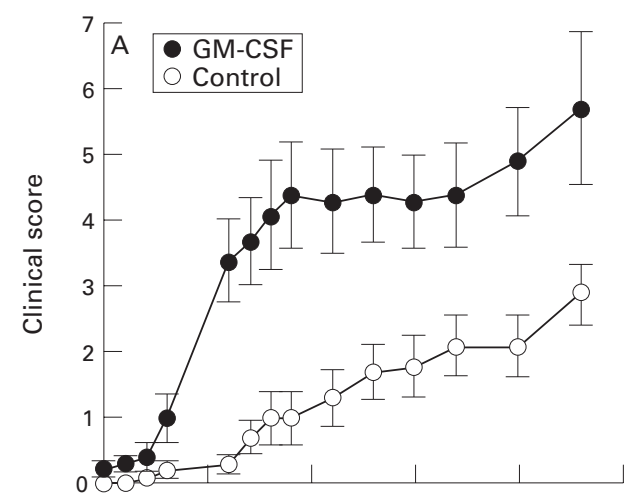

GM-CSF exhibited a more severe form of arthritis than the vehicle treated control animals in each of three separate experiments, as evidenced by the higher average clinical scores (table 1). Analysis of data pooled from the three experiments showed that GM-CSF treated mice exhibited a greater incidence of disease, with 25 of 28 mice showing clinical signs of arthritis compared with 18 of 30 control mice $(\mathrm{p}<0.05)$. The GM-CSF treated mice also displayed an earlier onset of disease with a mean (SEM) onset of 26.0 (0.6) days compared with 30.3 (1.3) days for control mice $(\mathrm{p}<0.01)$.

KINETICS OF GM-CSF EFFECT

A good correlation was seen between the means of the clinical scores and the rear paw size calliper measurements when the kinetics of the response to GM-CSF was examined (fig 1 (A) and (B), respectively). The administration of GM-CSF caused a rapid exacerbation of arthritis, which was already evident on the last day of the injection schedule (day 23) in the experiment in figure 1.

\section{HISTOLOGICAL ANALYSIS OF GM-CSF EFFECT ON} CIA

As confirmation that the response was indeed arthritis and not simply inflammation, at termination of the experiments the animals were killed, all limbs removed (whether clinically arthritic or not), and the joints fixed, decalcified, embedded in paraffin wax, and sections cut and stained with haematoxylin and eosin or toluidine blue for histological assessment. Figure 2 shows a representative frontal section of a knee joint taken from a GM-CSF treated mouse, stained with toluidine blue. The pathology is consistent with that seen in severe conventional CIA where a secondary immunisation is given, namely, loss of proteoglycan staining, cartilage destruction, synovitis, synovial hyperplasia, and pannus formation invading into the subchondral bone. ${ }^{10}$ Under the conditions of the CIA model used in this study (that is, no secondary CII injection), joint sections from vehicle treated mice rarely exhibited this degree of inflammation and tissue destruction.

To allow a quantitative comparison to be made between the joints of vehicle and GM-CSF treated mice a histological scoring system was adopted. Figure 3 shows the results of histological scoring of four different parameters of joint pathology (inflammation, pannus formation, cartilage damage, and bone damage) for GM-CSF versus control mice

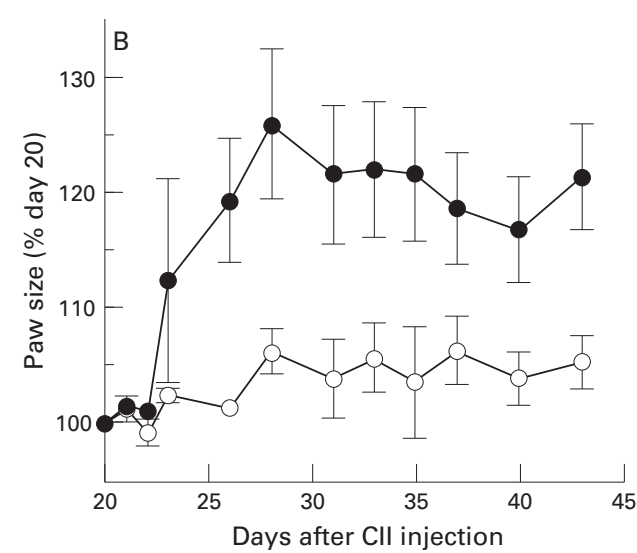

Figure 1 Kinetics of the effect of GM-CSF on clinical score and paw swelling in CIA. Mice were immunised with chick CII and 20 to 23 days later (experiment 2 from table 1) were given daily ip injections of either $15 \mu \mathrm{g}$ GM-CSF or vehicle (control). Arthritis development was assessed every two to three days by $(A)$ a clinical score (maximum score 12 per mouse) and (B) by calliper measurement of the hind footpad thickness. Results show the means (SEM) for 10 mice per group. For (B) the paw sizes of each individual limb were expressed as a percentage of their day 20 values and the mean (SEM) were then obtained for the 20 limbs.

Figure 2 Medial aspect of knee joint from type II collagen immunised mouse treated at days 21 to 25 by ip injection of loss of proteoglycan from the tibial articular cartilage is evident by the loss of intense staining (arrow) while the adjacent femoral cartilage is eroded down to the calcified cartilage layer (darker stain). The synovium (arrow head) is markedly thickened as a result of infiltration by inflammatory cells and proliferation of synovial cells and has invaded into the bone. Toluidine blue, frontal section, original magnification $\times 100$.

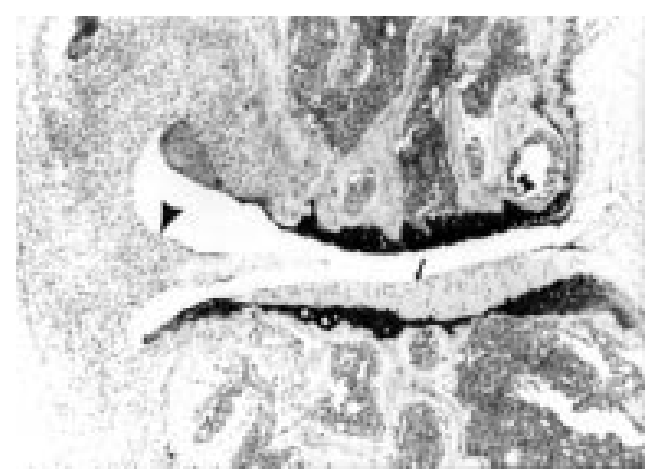
$15 \mu \mathrm{g}$ GM-CSF (experiment 1 from table 1). Pronounced 
earlier clinical assessment. The lesions of vehicle treated mice were sporadic and minimal to mild, whereas those from mice treated with GM-CSF were moderate to marked in severity.

\section{Discussion}

CIA is proving a valuable model for identification of the inflammatory mediators potentially involved in human RA. The approaches of either direct administration of cytokines (for example, IL1), ${ }^{12}$ of neutralising antibodies to cytokines (for example, to TNF $\alpha$ ), ${ }^{11}$ or of specific cytokine antagonists ${ }^{14} 15$ have in many instances shown a dramatic alteration in the severity or course of disease progression, or both, thereby implicating particular cytokines in the pathogenesis of joint disease. In this study we have used the first of these approaches to focus on the involvement of GM-CSF, a cytokine traditionally associated with haemopoiesis, although it can also have proinflammatory and proimmune actions. ${ }^{13}$ For this purpose, by omitting a secondary CII injection, we have established the CIA model under suboptimal response conditions to show an enhancing effect of GM-CSF.

We found that ip injection of GM-CSF, at three weeks after immunisation with CII, resulted in an exacerbation of arthritis symptoms. Over the time course of this study (up to 58 days after immunisation) mice treated with GM-CSF were more likely to develop arthritis and exhibited higher clinical scores, earlier clinical onset of disease, larger joint swelling measurements and increased histological scores for joint destruction than control animals receiving vehicle alone. At the level of histology, the GM-CSF exacerbated CIA manifested itself as a severe form of

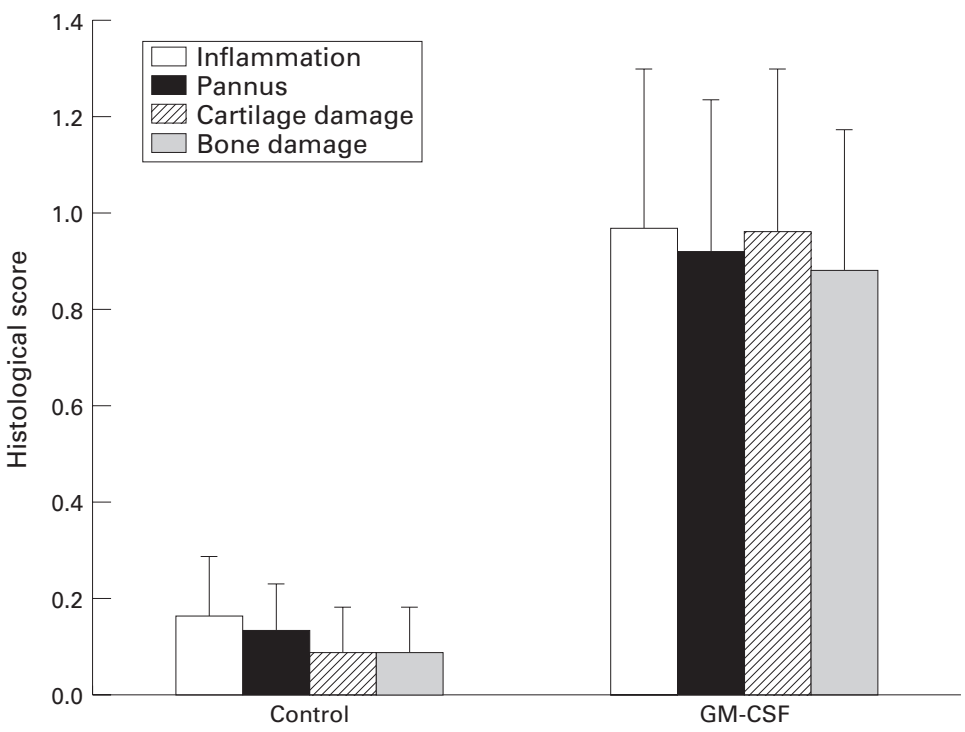

Figure 3 Histological assessment of CIA in mice treated with and without GM-CSF: Mice were immunised with chick CII and 21 to 25 days later were given daily ip injections of either $15 \mu \mathrm{g} G M-C S F$ or vehicle (control). At 45 days after immunisation the mice were killed, their limbs removed, and the joints (fore paw, knee, ankle, and hind paw) were fixed, decalcified, embedded in paraffin wax, and $5 \mu \mathrm{m}$ sections prepared and stained with haematoxylin and eosin. The joints were evaluated for the histological changes of inflammation, pannus formation, cartilage damage, and bone damage, each on a scale of 0 to 5. Results express the mean (SEM) for 10 mice per group. Foints from GM-CSF treated mice scored significantly higher $(p<0.05$, Mann-Whitney two sample test) than the control for each parameter. conventional CIA (where a secondary CII injection is given) with invasive inflammatory cells in the joint space, synovial hyperplasia, and invasion into sites of cartilage and bone degradation. Immunohistochemical studies are required to discover whether or not the administration of GM-CSF elicits a unique inflammatory response in this model, perhaps generating a different spectrum of effector cells to those usually observed in CIA.

The mechanism by which GM-CSF exacerbates CIA has not been elucidated in this study and awaits further investigation; however, based on its known in vitro and in vivo actions, it probably involves modulation of the levels and functions of circulating and/or activated monocyte-macrophages and/or neutrophils. Any of the numerous in vitro actions of GM-CSF detailed above could be contributing mechanisms. Studies in vivo have shown that thrice daily ip injections of GM-CSF $(0.2 \mu \mathrm{g})$ for six days elicits a twofold increase in both circulating neutrophils and in spleen monocytes in BALB/c, C57BL, and $\mathrm{C} 3 \mathrm{H} / \mathrm{HeJ}$ mice. ${ }^{16}$ Increased numbers of neutrophils, monocytes (major cell type), and eosinophils were also described in the peritoneal cavity, the macrophages of which exhibited an enhanced phagocytic capacity, indicating activation. ${ }^{16}$ In this study, the daily administration of $15 \mu \mathrm{g}$ GM-CSF probably had similar effects on at least some of these cell populations, although the mice concerned have been sensitised to develop inflammatory autoimmune arthritis.

GM-CSF, when coadministered subcutaneously or intradermally at the antigenic site, has been recognised as an effective adjuvant for increasing cellular and humoral immunity to peptide/protein antigens. ${ }^{17}$ Although the 'professional' antigen presenting cells, the dendritic cells, are generated from bone marrow cells in vitro in response to GM-CSF, ${ }^{18}$ these cells lack the ability to present collagen to $\mathrm{T}$ cells. ${ }^{19}$ As both macrophages and granulocytes feature in the joints of mice with CIA ${ }^{10}$ then either or both cell types may be the target of GM-CSF in this study. GM-CSF may have affected these cells through local or systemic actions, or both; locally, through the 'activation' of cells already within the joints as part of the ongoing immune reaction to produce inflammatory mediators leading to more severe joint damage ${ }^{9}$; and systemically, by availing greater numbers of circulating activated cells ${ }^{16}$ for migration into the inflamed joint.

Finally, although this study has shown that exogenously added GM-CSF promotes a severe form of CIA, this does not indicate whether it is a requisite component of the 'normal' pathogenesis of CIA and so further studies using neutralising antibodies to GM-CSF are currently underway to consider this. Nevertheless, these findings are consistent with the proinflammatory actions of GM-CSF in exacerbating human $\mathrm{RA}^{6}$ and highlight the important role GM-CSF could have in determining the progression and severity of inflammatory joint disease. 
This work was supported by a Program Grant from the National Health and Medical Research Council of Australia and grants from the Arthritis Foundation of Australia and Amgen.

1 Metcalf D. The Florey Lecture, 1991. The colonystimulating factors: discovery to clinical use. Philos Trans $R$ stimulating factors: discovery to

2 Metcalf D, Elliott M J, Nicola N A. The excess numbers of peritoneal macrophages in granulocyte-macrophage colony-stimulating factor transgenic mice are generated by local proliferation. J Exp Med 1992;175:877-84.

3 Xu W D, Firestein G S, Taetle R, Kaushansky K, Zvaifler N J. Cytokines in chronic inflammatory arthritis. II. rheumatoid synovial effusions. J Clin Invest 1989;83:87682 .

4 Berenbaum F, Rajzbaum G, Amor B, Toubert A. Evidence for GM-CSF receptor expression in synovial tissue. An analysis by semi-quantitative polymerase chain reaction on rheumatoid arthritis and osteoarthritis synovial biopsies. Eur Cytokine Netw 1994;5:43-6.

5 Field M, Clinton L. Expression of GM-CSF receptor in

6 Hazenberg B P C, Van Leeuwen M A, Van Rijswijk M H, Stern A C, Vellenga E. Correction of granulocytopenia in Felty's syndrome by granulocyte-macrophage colonystimulating factor. Simultaneous induction of interleukin-6 release and flare-up of the arthritis. Blood 1989;74:2769-73.

7 Campbell I K, Novak U, Cebon J, Layton J E, Hamilton J A. Human articular cartilage and chondrocytes produce hemopoietic colony-stimulating factors in culture in response to IL-1. J Immunol 1991;147:1238-46.

8 Leizer T, Cebon J, Layton J E, Hamilton J A. Cytokine regulation of colony-stimulating factor production in cultured human synovial fibroblasts. 1. Induction of GM-CSF and G-CSF production by interleukin-1 and tumor necrosis factor. Blood 1990;76:1989-96.

9 Hamilton J A. Rheumatoid arthritis: Opposing actions of haemopoietic growth factors and slow-acting antirheumatic drugs. Lancet 1993;342:536-9.
10 Staines N A, Wooley P H. Collagen arthritis - what can it teach us? Br J Rheumatol 1994;33:798-807.

11 Williams R O, Feldmann M, Maini R N. Anti-tumor necrosis factor ameliorates joint disease in murine collageninduced arthritis. Proc Natl Acad Sci USA 1992; 89:9784-8.

12 Hom J T, Bendele A M, Carlson D G. In vivo administration with IL-1 accelerates the development of collagen-induced arthritis. J Immunol 1988;141:834-41.

13 Hamilton J A, Stanley E R, Burgess A W, Shadduck R K. Stimulation of macrophage plasminogen activator activity by colony-stimulating factors. J Cell Physiol 1980; by colony-stim
103:435-45.

14 Wooley P H, Whalen J D, Chapman D L, Berger A E, Richard K A, Aspar D G, et al. The effect of an interleukin-1 receptor antagonist protein on type II collagen-induced arthritis and antigen-induced arthritis in mice. Arthritis Rheum 1993;36:1305-14.

15 Wooley P H, Dutcher J, Widmer M B, Gillis S. Influence of a recombinant human soluble tumor necrosis factor receptor FC fusion protein on type II collagen-induced arthritis in mice. J Immunol 1993;151:6602-7.

16 Metcalf D, Begley C G, Williamson D J, Nice E C, De Lamarter J, Mermod J -J, et al. Hemopoietic responses in mice injected with purified recombinant murine GMCSF. Exp Hematol 1987;15:1-9.

17 Disis M L, Bernhard H, Shiota F M, Hand S L, Gralow J R, Huseby E S, et al. Granulocyte-macrophage colonystimulating factor: an effective adjuvant for protein and peptide-based vaccines. Blood 1996;88:202-10.

18 Inaba $\mathrm{K}$, Inaba $\mathrm{M}$, Romani $\mathrm{N}$, Aya $\mathrm{H}$, Deguchi $\mathrm{M}$, Ikehara $\mathrm{S}$, et al. Generation of large numbers of dendritic cells from mouse bone marrow cultures supplemented with granulocyte/macrophage colony-stimulating factor. J Exp Med 1992;176:1693-702.

19 Michaëlsson E, Holmdahl M, Engström Å, Burkhardt H, Scheynius A, Holmdahl R. Macrophages, but not dendritic cells, present collagen to T cells. Eur J Immunol 1995;25:2234-41. 